\title{
COMBINATION OF TERRASAR-X AND OPTICAL IMAGERY FOR LU/LC MAPPING USING AN OBJECT-BASED APPROACH
}

\author{
J.A. Recio ${ }^{\text {a }}$ L.A. Ruiz, T. Hermosilla ${ }^{\text {a }}$ V. Herrera-Cruz , A. Fernández-Sarría ${ }^{a}$

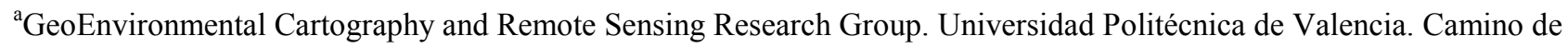 \\ Vera s/n, 46022 Valencia, Spain. -jrecio@cgf.upv.es \\ b Infoterra GmbH. 88039 Friedrichshafen, Germany. - Virginia.Herrera-Cruz@astrium.eads.net
}

KEY WORDS: LU/LC mapping; descriptive feature extraction; object-oriented classification; Landsat, TerraSAR-X.

\begin{abstract}
:
In this study, the impact of the use of backscattering intensity and texture features obtained from TerraSAR-X images for LULC classification of agricultural and forest areas, and its combination with features extracted from Landsat 7 EMT+ optical imagery is analyzed. The performance of texture descriptors on radar images is evaluated. After data pre-processing and the definition of classes in the study area, every object is described by means of a set of features computed from the TerraSAR-X and optical imagery, using a plot-based approach. Cadastral cartographic limits are employed for objects definition. Next, objects are classified using decision trees combined with boosting techniques. The classification results are compared to the LULC contained in the testing database, and the errors evaluated in terms of the different groups of variables, the source of data used, and their performance for the variety of classes considered. The classification results bring some possibilities and limitations of combining features from optical and radar imagery, evidence the complementary information provided by both types of data to face these applications.
\end{abstract}

\section{INTRODUCTION}

The demand of updated geographic data is constantly increasing, due to changes in the landscape and the influence of these changes on the decisions affecting management of the territory. Object-based image classification techniques are being incorporated in Land-Use/Land-Cover (LULC) geospatial database updating processes. High frequency and massive data acquisition generates high volume of information that needs to be efficiently processed to be used in different applications, and object-based techniques are efficient combining multisensor data, since they significantly reduce the data to be processed. The segmentation method employed is key in the descriptive features that are derived from objects to be used in the classification, since these will differ depending on the algorithm and selected parameters. Plot-based image classification is a particular object-based classification case that uses cartographical limits to create objects. These limits better enable the definition of significant objects in the real world than automatic pixel aggregation. This is an especially suitable methodology for anthropogenic environments such as agricultural areas. The use of cartographic limits to perform the image segmentation enables to easily relate the information derived from the imagery with the LULC geospatial databases.

Landsat imagery has been used in multiple researches and analysis for change detection and LULC classification (Bauer et al., 2003). In spite of the medium spatial resolution (30 meters), Landsat data present seven spectral bands (plus one panchromatic) and high acquisition frequency (16 days). This enables to periodically monitor the LULC of a territory.

A radar system is an active sensor, illuminating a ground target area with its own energy signal. Radar surface feature interaction, or scattering, and the characteristics of this scattered energy, or backscatter, are dependent upon the geometric and electrical factors of the ground target area. Such factors include target material, orientation, moisture content and the degree of surface roughness (Herold et al., 2004). Radar image backscatter is often a direct result of the ground surface texture (Dobson et al., 1995). Radar data have been used solely or combined with optical imagery in the literature for land use/land cover classification and change detection tasks in several scenarios such as agricultural areas (Nizalapur, 2008; Zou et al., 2010), forest (Dutra et al., 1998; Milne et al., 2000), or urban environments (Del Frate et al., 2008; Yang et al, 2009). Most of the analyses concerning radar data have been carried out using a pixel-based approach. However, some authors (Hong et al., 2007; Liu et al., 2008; Qi et al., 2010) have explored the possibilities of object-based image analysis approaches, where image objects are defined using automatic segmentation methods.

This study evaluates the effect on the classification accuracy of combining Landsat 7 ETM+ imagery with backscattering data acquired with TerraSAR-X, using a plot-based approach. Plotbased image classification is performed with decision trees together with boosting multiclassifier method. Errors are evaluated in terms of the different groups of descriptive features, source of data used, and their performance for the classes defined. The study area is an agricultural zone mixed with forest and shrublands located in the northwest of Spain.

\section{STUDY AREA AND DATA}

The study area was defined in the local administrative area of A Limia, located in the region of Galicia (Spain). The landscape is characterized by a broad combination of sparse villages and rural settlements, diverse agriculture, forest and shrubland. Several tests have been carried out in this area to assess the classification performance when considering high spatial 
resolution imagery (Hermosilla et al., 2010a) combined with geographic ancillary information (Recio et al., 2010) or LiDAR data (Hermosilla et al., 2010b), and high spatial resolution multispectral imagery (Ruiz et al., 2011).

Cartographic boundaries were used to define the analysis objects (plots), and were obtained from the Spanish Land Parcel Identification System (SIGPAC), a National geospatial database for agriculture management. The plots represent a continuous area of land within a parcel for a single agricultural use.

RADAR imagery has $3 \mathrm{~m} /$ pixel spatial resolution and was acquired by TerraSAR-X on October $27^{\text {th }}, 2008$. With its active phased array X-band SAR antenna (wavelength $31 \mathrm{~mm}$, frequency $9.6 \mathrm{GHz}$ ), TerraSAR-X acquires new high-quality RADAR images, circling Earth in a polar orbit at $514 \mathrm{~km}$ altitude. The orbit is selected such that the satellite follows a sun-synchronous orbit. TerraSAR-X is designed to carry out its task independently of weather conditions and illumination.

Multispectral image data were acquired with Landsat 7 Enhanced Thematic Mapper Plus (ETM + ) on December $23^{\text {rd }}$ of 2008. Bands $1(0.45-0.515 \mu \mathrm{m}), 2(0.525-0.605 \mu \mathrm{m}), 3(0.63-$ $0.69 \mu \mathrm{m}), 4(0.75-0.90 \mu \mathrm{m}), 5(1.55-1.75 \mu \mathrm{m})$ and $7(2.09-2.35$ $\mu \mathrm{m})$ were considered in the analysis. These bands have a spatial resolution of $30 \mathrm{~m} /$ pixel. A detail of the data employed in the study area is shown in Figure 1.
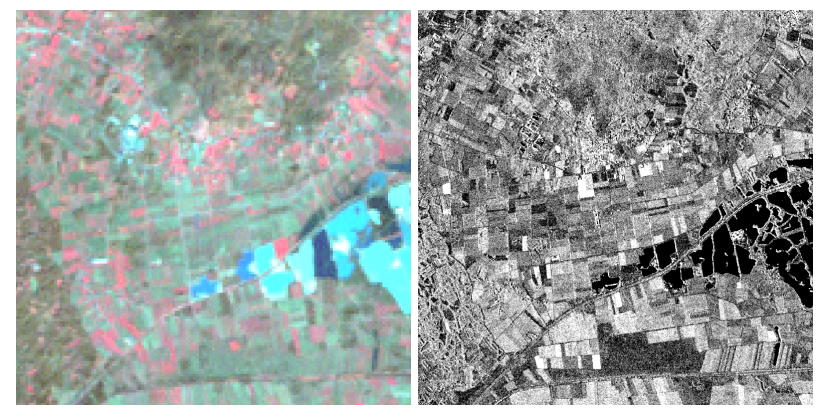

Figure 1. Study area images in Landsat 7 ETM+ colour infrared composition (left) and TerraSAR-X (right).

Five simple classes were defined: forest, shrublands, irrigated crops, arable lands, and urban areas. Using photointerpretation techniques and field information, around 50 training samples per class were selected avoiding the missed strips produced due to the scan line corrector failure in the ETM+.

\section{METHODOLOGY}

\subsection{TerraSAR-X data preprocessing}

The TerraSAR-X image was acquired in the Stripmap mode in single polarization $\mathrm{HH}$. It was provided in the Enhanced Ellipsoid Corrected mode (EEC). In this mode, the image is projected and re-sampled to the WGS84 reference ellipsoid and the distortions caused by varying terrain height are corrected using an external DEM (Fritz and Eineder, 2009). The pixel localization in these products is usually very accurate, depending the final accuracy on the type of terrain as well as the quality and resolution of the DEM and the incidence angle.
Prior to descriptive feature extraction process, the radiometric calibration of TerraSAR-X image was done. A full description of the employed method is in Infoterra (2008). Firstly, the values of radar brightness or Beta Naught $\left(\beta^{0}\right)$ are computed according to the equation (1):

$$
\beta_{d B}^{0}=10 \% \log _{10}\left(k_{s} \cdot|D N|^{2}\right)
$$

where $k_{s}$ is the calibration factor given in the TerraSAR-X data delivery package annotation file, and $D N$ is the digital number given in the image product. The values of the radar reflectivity per unit area in the ground range, known as Sigma Naught $\left(\sigma^{0}\right)$, are derived from Beta Naught values taking into account the local incident angle, according to the equation (2):

$$
\sigma_{d B}^{0}=\beta_{d B}^{0}+10 \log _{10}\left(\sin \theta_{l o c}\right)
$$

where $\theta_{l o c}$ is the local incidence angle between the radar beam and the normal to the illuminated surface. This value is provided for each pixel in the Geocoded Incidence angle Mask (GIM).

\subsection{Descriptive feature extraction}

Descriptive features were computed using the FETEX 2.0 object-based image analysis software (Ruiz et al., in press). Spectral features were computed from Landsat 7 ETM+ imagery. These features are particularly useful in the characterization of spectrally homogeneous classes, such as herbaceous crops or fallow fields. Mean and standard deviation values were computed from the six spectral bands considered, and also from the Normalized Difference Vegetation Index (NDVI), calculated by using the red and the near infrared bands.

A set of texture descriptive features were extracted from the TerraSAR-X image. Texture information may be as important as spectral in radar images since the backscattering depends on the roughness of the surface and this can be captured using the values of the neighbourhood of pixels. Thus, texture features quantify the spatial distribution of the intensity values in the analysed objects, providing additional information useful to characterize the LULC (Ruiz et al., 2004). A considerable number of quantitative texture features and approaches have been reported using different methodologies, that are traditionally computed considering the neighbourhood of each pixel on the image. In this study, each texture feature value is referred to a particular object, since it is extracted from each group of pixels that constitute an object. Texture features are: edgeness factor, first order histogram descriptors, grey level cooccurrence matrix (GLCM) and wavelet transform derived variables.

The Edgeness factor represents the density of edges present in a neighbourhood (Laws, 1985), in our case, it is determined by the limits of the object itself, and was characterized by using the mean and the standard deviation values of the edgeness factor inside each plot First order texture descriptors, skewness and kurtosis, are directly related to the shape and distribution of the frequency histogram of an object. 
The following GLCM based features (Haralick et al., 1973) were computed: contrast, uniformity, entropy, variance, covariance or product moment, inverse difference moment and correlation. Since an object-oriented approach is used, only one GLCM is computed for each object, describing the cooccurrences of the pixel values that are separated at a distance of one pixel inside the plot, and considering the average value of four principal orientations $\left(0^{\circ}, 45^{\circ}, 90^{\circ}\right.$ and $\left.135^{\circ}\right)$. Figure 2 shows some examples of GLCM computed for plots with the defined classes.

A group of texture features was derived by applying the wavelet transform to the TerraSAR-X image. The wavelet transform allows for the decomposition of a signal using a series of elemental functions called wavelets and scaling, which are created by the scaling and translation of a base function, known as the mother wavelet. Since the most relevant texture information is lost in the lowpass filtering process, only fluctuations are used to calculate texture descriptors. If the inverse transform is applied to the fluctuations, three reconstructed images, or details, are obtained: horizontal, vertical and diagonal. This process is called multiresolution analysis. Different texture features have been extracted from wavelet details or fluctuations using the Coiflet wavelet with a support of 6 pixels. A total of eight Haralick's features derived from the GLCM were extracted from the image containing the sum of the reconstructed details (mean, contrast, uniformity, entropy, variance, covariance, inverse difference moment, and correlation), as well as the mean and standard deviation of the edgeness factor. In order to avoid the analysis of pixels located out of the considered objet, an erosion filter using a circular structuring element with a diameter size equal to the support of the wavelet function was applied to the final image.

\subsection{Classification and accuracy assessment}

Three classifications were performed, considering (i) spectral information derived from Landsat 7 ETM+ imagery, (ii) texture information computed using TerraSAR-X imagery, and (iii) combining both information sources. Plots were classified by applying the decision-trees built with the C5.0 algorithm (Quinlan, 1993). This algorithm searches the features that best separate one class from the others by using mutually exclusive conditions, until homogeneous subgroups are generated, i.e. all the elements in a subgroup belong to the same class, or a stopping condition is satisfied. In addition, a boosting multiclassifier method was used, based on the assignment of weights to the training samples, enabling an increase of the classification accuracy.

Classification accuracy assessment was based on the analysis of the confusion matrix (Congalton, 1991), by comparing the class assigned to each evaluation sample with the information contained in the reference database. The overall accuracies of the classifications were computed, as well as the producer and user accuracies for each class, revealing the errors of omission and commission respectively. Since the collection of significant training samples was a difficult task, and a representative set of testing data was needed to ensure a correct evaluation, the leave-one-out cross-validation technique was used. This method is based on using a single observation from the original sample as the validation data, and the remaining observations as the training data. This is repeated such that each observation in the sample is used once as the validation data.
In addition to the classification accuracy assessment, a study of the relationships between the descriptive features and their specific contribution to the classification accuracy was performed. Statistical linear discriminant analysis was used to determine the significance of the features for the particular classification problem.
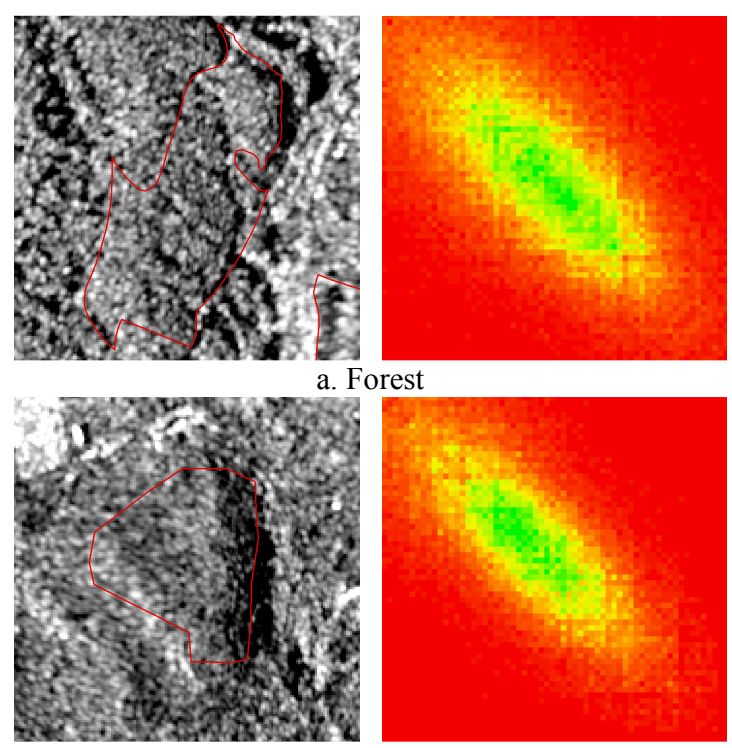

b. Shrublands
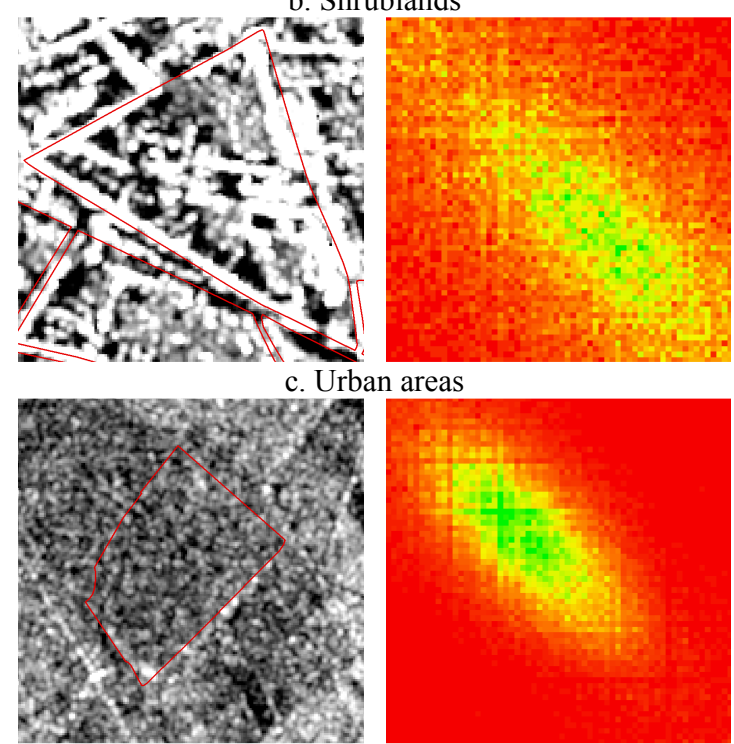

d. Irrigated crops

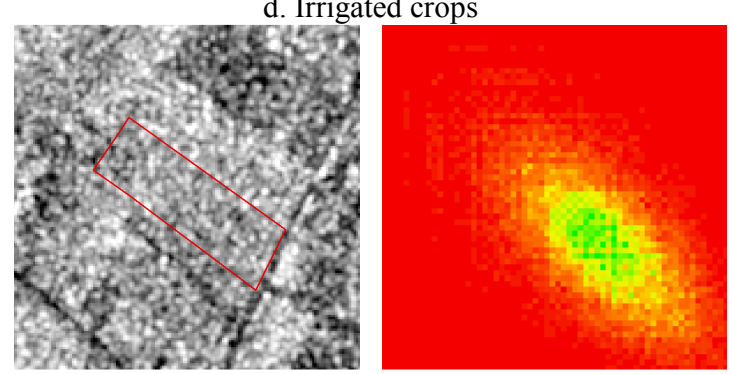

e. Arable lands

Co-occurrence frequency: $\min \quad \max$

Figure 2. Examples of parcels of the defined classes in TerraSAR-X (left) and their graphic representation of the GLCM computed per-object (right). 


\section{RESULTS}

The results of the first classification performed using Landsat 7 ETM+ imagery-derived spectral descriptive features are shown in the confusion matrix presented in the Table 1 . Overall classification accuracy reaches a value of $84.6 \%$ for the five simple classes considered. Highest user's and producer's accuracy values are obtained for irrigated crops class, which barely presents misclassifications. Arable lands plots are classified with a high rate of success, presenting some confusions with urban class. This class also presents a good performance, with user's and producer's accuracies values higher than $80 \%$. The classes forest and shrublands have the lowest classification performance and elevated mutual confusion. This is due to the limitations presented by exclusively considering spectral features to describe the objects (plots).

\begin{tabular}{|c|c|c|c|c|c|c|}
\hline & \multicolumn{5}{|c|}{ Reference } & \multirow{2}{*}{ 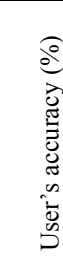 } \\
\hline & $\begin{array}{l}\overrightarrow{0} \\
\stackrel{0}{0} \\
\text { con }\end{array}$ & 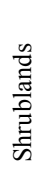 & 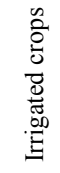 & 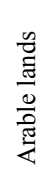 & $\begin{array}{l}\text { 呡 } \\
\text { 营 }\end{array}$ & \\
\hline Forest & 40 & 10 & & & 2 & 77 \\
\hline Shrublands & 10 & 36 & & 1 & 3 & 72 \\
\hline Irrigated crops & & & 49 & & 1 & 98 \\
\hline Arable lands & & & & 44 & 4 & 92 \\
\hline Urban & & 4 & & 4 & 45 & 85 \\
\hline Producer's acc. (\%) & 80 & 72 & 100 & 90 & 82 & 84.6 \\
\hline
\end{tabular}

Table 1. Confusion matrix of the classification using spectral features from Landsat $7 \mathrm{ETM}+$ data.

When only texture information extracted from TerraSAR-X imagery is considered the classification overall accuracy obtained is $75.4 \%$, as it is shown in the confusion matrix of Table 2. The distribution of errors radically changes regarding to the Landsat $7 \mathrm{ETM}+$ data. Urban class plots are almost perfectly classified. The rest of classes present many confusions. There are especially noticeable misclassifications in the case of irrigated crops and arable lands, producing the lowest values in the user's and producer's accuracy indices. Shrublands class attains accuracy values that are comparable to those reached considering only Landsat 7 ETM+ imagery, but errors are distributed among several classes. Besides, the use of textures derived from TerraSAR-X imagery produces a slightly increase on the accuracy of forest class, significantly reducing its confusion with shrublands class.

\begin{tabular}{|c|c|c|c|c|c|c|}
\hline & \multicolumn{5}{|c|}{ Reference } & \multirow{2}{*}{ 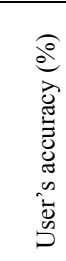 } \\
\hline & 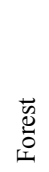 & $\frac{\frac{D}{0}}{\frac{\vec{E}}{0}}$ & 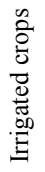 & $\begin{array}{l}\frac{n}{0} \\
\frac{\pi}{0} \\
\frac{0}{0} \\
\frac{\pi}{2}\end{array}$ & 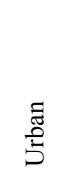 & \\
\hline Forest & 44 & 3 & 4 & 2 & & 83 \\
\hline Shrublands & 2 & 35 & 6 & 6 & & 71 \\
\hline Irrigated crops & 1 & 6 & 25 & 9 & & 61 \\
\hline Arable lands & 2 & 6 & 14 & 32 & & 59 \\
\hline Urban & 1 & & & & 55 & 98 \\
\hline Producer's acc. (\%) & 88 & 70 & 51 & 65 & 100 & 75.4 \\
\hline
\end{tabular}

Table 2. Confusion matrix of the classification using texture features from TerraSAR-X data.
The combination of spectral features computed from Landsat 7 ETM+ imagery with texture features extracted from TerraSAR$\mathrm{X}$ data produces a synergic effect that significantly improves the classification results (see confusion matrix in Table 3), reaching an overall accuracy value of $95.3 \%$. User's and producer's accuracy values are more balanced and higher than $90 \%$ for almost all classes. Main misclassifications are produced in forest and shrublands, with some mutual confusions and minor errors with other classes. Object-based image analysis enables the combination of both data with different spatial resolution.

\begin{tabular}{|c|c|c|c|c|c|c|}
\hline & \multicolumn{5}{|c|}{ Reference } & \multirow{2}{*}{ 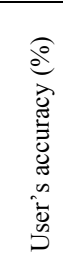 } \\
\hline & 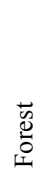 & $\begin{array}{l}\frac{n}{0} \\
\frac{\vec{E}}{0} \\
\text { 吾 }\end{array}$ & 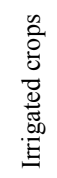 & $\begin{array}{l}\frac{n}{0} \\
\frac{\pi}{\pi} \\
\frac{0}{0} \\
\frac{\pi}{4}\end{array}$ & $\begin{array}{l}\text { : } \\
\text { 옴 }\end{array}$ & \\
\hline Forest & 45 & 4 & & & & 92 \\
\hline Shrublands & 3 & 44 & & 1 & & 92 \\
\hline Irrigated crops & & 1 & 49 & & & 98 \\
\hline Arable lands & & 1 & & 48 & & 98 \\
\hline Urban & 2 & & & & 55 & 96 \\
\hline Producer's acc. (\%) & 90 & 88 & 100 & 98 & 100 & 95.3 \\
\hline
\end{tabular}

Table 3. Confusion matrix of the classification combining Landsat $7 \mathrm{ETM}+$ and TerraSAR-X data.

The specific contribution of the descriptive features in the classification model computed by means of linear discriminant analysis is shown in Figure 3. The first variable included in the model, i.e. the most discriminative, is the edgeness factor standard deviation computed from TerraSAR-X. The single addition into the model of this descriptive feature produces a classification overall accuracy higher than $50 \%$. Afterwards, the following most discriminate variables are three spectral features derived from Landsat 7 ETM + the means of the NDVI, $4^{\text {th }}$ and $3^{\text {rd }}$ bands.

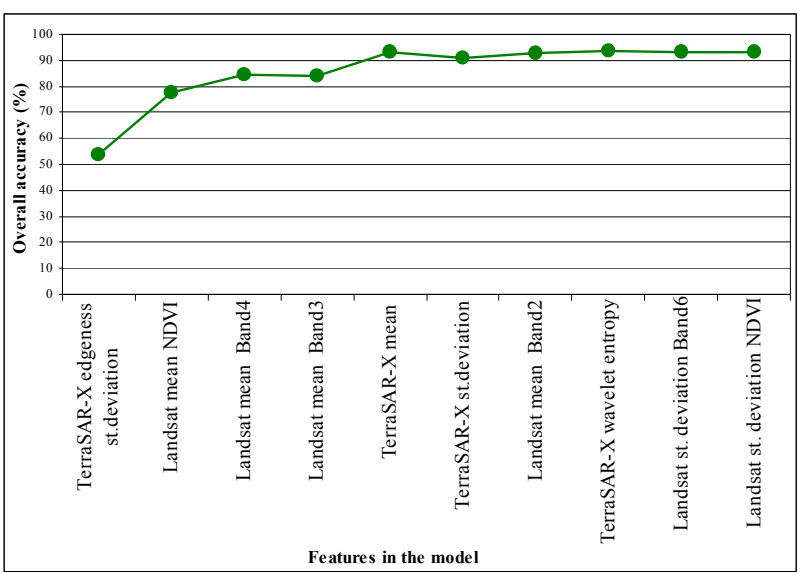

Figure 3. Evolution of the estimated overall accuracy as new descriptives features are introduced into the discriminant model when Landsat 7 ETM+ and TerraSAR-X data are combined.

Using only these features, the overall accuracy reaches a value close to $85 \%$. The subsequent feature added to the discriminant model is again a texture feature, corresponding to the mean 
value of the object computed from the TerraSAR-X data, which increases the overall accuracy up to $93 \%$. The addition of new descriptive features has no significant effect on the overall accuracy value. The results of this analysis illustrate the complementary nature and effect of the proposed descriptive features, as well as the possibility of increasing the efficiency of the classification in terms of accuracy and reducing the number of variables by using only a selected and highly discriminant group of features.

\section{CONCLUSIONS}

This paper presents and analyzes a methodology for LULC classification using generic classes and a plot-based classification approach using Landsat 7 ETM+ imagery and TerraSAR-X imagery. The classification was performed using decision trees and combining different descriptive features. Object-based image analysis facilitates the combination of radar and middle resolution imagery, since they are not required to have the same pixel size, and the border effect characteristic of the texture analysis is avoided. Spectral descriptive features derived from optical images - and texture descriptive features derived from radar images - are complementary, enabling a correct description of land cover types with homogeneous or heterogeneous structures. The proposed methodology provides the means for reducing the updating periods in land parcel identification systems obtaining accurate information with reduced costs.

\section{ACKNOWLEDGEMENTS}

The authors appreciate the support from Infoterra $G m b H$ for providing the TerraSAR-X imagery, and BANTEGAL, Xunta de Galicia, for providing the cartography and field sample data, and the financial support provided by the Spanish Ministry of Science and Innovation in the framework of the project CGL2010-19591/BTE.

\section{REFERENCES}

Bauer M., Yuan F., Saway K., 2003. Multi-Temporal Landsat Image Classification and Change Analysis of Land cover in the Twin Cities (Minnesota) Metropolitan Area. Workshop on the analysis of multi-temporal remote sensing images, Ispra, Italy, pp. 368-375.

Congalton, R., 1991. A review of assessing the accuracy of classications of remotely sensed data. Remote Sensing of Environment, 37(1), pp. 35-46.

Del Frate, F., Pacifici, F., Solimini, D., 2008. Monitoring Urban Land Cover in Rome, Italy, and Its Changes by SinglePolarization Multitemporal SAR Images. IEEE Journal of Selected Topics in Applied Earth Observations and Remote Sensing, 1(2), pp.87-97.

Dobson, M.C., Ulaby, F.T., Pierce, L.E., 1995. Land cover classification and estimation of terrain attributes using synthetic aperture radar. Remote Sensing of Environment, 51, pp. 199214.

Dutra, L.V., Huber, R., Hernandez Filho, P., 1998. Primary forest and land cover contextual classification using JERS-1 data in Amazonia, Brazil. Proceedings of the International Geoscience and Remote Sensing Symposium, Seattle, USA, Vol 5. pp. $2743-2745$.

Fritz, T., Eineder, M., 2009. TerraSAR-X Ground Segment Basic Product Specification Document, TX-GS-DD-3302, Issue 1.6, (online). http://www.infoterra.de/asset/cms/file/tx-gs-dd3302_basic-product-specification-

document 1.6_signed_small.pdf (accessed 14 April 2011)

Haralick, R.M., Shanmugam, K., Dinstein, I., 1973. Texture features for image classification. IEEE Transactions on Systems, Man and Cybernetics, 3(6), pp. 610-622.

Hermosilla, T., Díaz-Manso, J.M., Ruiz, L.A., Recio, J.A., Fernández-Sarría, A., Ferradáns-Nogueira, P., 2010a. Parcelbased image classification as a decision-making supporting tool for the Land Bank of Galicia (Spain). The International Archives of Photogrammetry, Remote Sensing and Spatial Information Sciences. Vol. XXXVIII (4-8-2-W9), pp. 40-45.

Hermosilla, T., Almonacid, J., Fernández-Sarría, A., Ruiz, L.A., Recio, J.A., 2010b. Combining features extracted from imagery and lidar data for object-oriented classification of forest areas. The International Archives of Photogrammetry, Remote Sensing and Spatial Information Sciences. Vol. XXXVIII (4/C7), 6p.

Herold, N., Haack, B., Solomon, E., 2004. An evaluation of radar texture for land use/cover extraction in varied landscapes. International Journal of Applied Earth Observation and Geoinformation, 5(2), pp. 113-128.

Hong, G., Y. Zhang, A. Zhang, F. Zhou, J. Li, 2007. Fusion of MODIS and Radarsat data for crop type classification - an initial study. The International Archives of Photogrammetry, Remote Sensing and Spatial Information Sciences, Urumchi, Xinjiang, China, Vol. XXXVI, Part 4/W54, pp. 153-156.

INFOTERRA, 2008. Radiometric Calibration of TerraSAR-X Data, (online) http://www.infoterra.de/asset/cms/file/tsxx-itd-tn0049-radiometric_calculations_i1.00.pdf (accessed 14 April 2011)

Laws, K. I., 1985. Goal-directed texture image segmentation. Applications of Artificial Intelligence II, SPIE, 548, pp. 19-26.

Liu, J., Pattey, E., Nolin, M.C., 2008. Object-based Classification of High Resolution SAR Images for Within Field Homogeneous Zone Delineation. Photogrammetric Engineering \& Remote Sensing, 74(9), pp. 1159-1168.

Milne, A.K., Lucas, R.M., Cronin, N., Dong, Y., Witte, C., 2000. Forest and woodland biomass and classification using airborne and spaceborne radar data. The International Archives of Photogrammetry and Remote Sensing, Amsterdam, Netherlands, Vol. XXXIII, Part B7, pp. 876-885.

Nizalapur, V., 2008. Land cover classification using multisource data fusion of ENVISAT-ASAR and IRS P6 LISS-III satellite data - A case estudy over tropical moist deciduous forested regions of Karnataka, India. The International Archives of the Photogrammetry, Remote Sensing and Spatial Information Sciences, Beijing, China, Vol. XXXVII, Part B6b, pp. 329-334.

Qi, Z., Yeh, A.G.O., Zhang, X., 2010. Fusion of RADARSAT-2 PolSAR and PolInSAR Data for Land Use and Land Cover 
Classification. Proccedings of the Asia GIS 2010 International Conference. Kaohsiungm, Taiwand.

Quinlan, J.R., 1993. C4.5. Programs for machine learning. Morgan Kaufmann, San Mateo.

Recio, J.A., Hermosilla, T., Ruiz, L.A., Fernández-Sarría, A., 2010. Addition of geographic ancillary data for updating geospatial databases. The International Archives of Photogrammetry, Remote Sensing and Spatial Information Sciences, Haifa, Israel, Vol. XXXVIII, (4-8-2-W9), pp. 46-51.

Ruiz, L.A, Fernández-Sarria, A., Recio, J.A., 2004. Texture feature extraction for classification of remote sensing data using wavelet decomposition: A comparative study. The International Archives of Photogrammetry, Remote Sensing and Spatial Information Sciences, Vol. XXXV, Part B4, pp. 1109-1115.

Ruiz, L.A., Hermosilla, T., Serisa, G., Recio, J.A., FernándezSarría, A., 2011. A Multi-Approach and Object-Oriented Strategy for Updating LU/LC Geo-databases Based on Worldview-2 Imagery. Geospatial World Forum, Hyderabad, India.

Ruiz, L.A., Recio, J.A., Fernández-Sarría, A., Hermosilla, T., In press. A feature extraction software tool for agricultural objectbased image analysis. Computers and Electronics in Agriculture. http://dx.doi.org/10.1016/j.compag.2011.02.007

Yang, W., Zou, T.Y., Dai, D.X., Shuai, Y.M., 2009. Supervised land-cover classification of TerraSAR-X imagery over urban areas using extremely randomized forest. Proceedings of the Joint Urban Remote Sensing Event (JURSE '09), Shanghai, China.

Zou, T., Yang, W., Dai, D., Sun, H., 2010. Polarimetric SAR Image Classification Using Multifeatures Combination and Extremely Randomized Clustering Forests. EURASIP Journal on Advances in Signal Processing, Vol. 2010, Article ID 465612 . 